\title{
Tuning and characterization of Twente wiggler
}

\author{
J.W.J. Verschuur ${ }^{\mathrm{a} \cdot *}$, R.W. Warren ${ }^{\mathrm{b}}$ \\ "University of Twente, Department of Applied Physics, P.O. Box 217. 7500 AE Enschede, Netherlands \\ 'Los Alamos National Laboratory, Los Alamos, NM 87545, USA
}

\begin{abstract}
For correcting field errors in the Twente wiggler small metal shims were placed on the pole faces. When applied to correct errors in the on-axis field a large increase in the errors in the off-axis field was found. When instead errors in the off-axis field are corrected, the errors in the on-axis field are reduced as well. Only small corrections were locally required to make the errors in the on-axis field acceptable. The focussing strength of the wiggler has been measured in both transverse planes and was found to lead to a slightly elliptical shape for a matched electron beam. Longitudinal phase errors have been measured for the Twente wiggler and are negligible.
\end{abstract}

\section{Arguments about the required precision}

After constructing a wiggler it is important to have some method to reduce field errors that are inevitably present. Since one can never fix all of the errors it is important to decide which errors are particularly important and how large each of these errors can be in order to be negligible. The starting points are the most important criteria: keep the longitudinal phase errors small, have approximately equal two plane focusing, and have good overlap of the electron beam and optical beam. The phase errors are in general small, and will be discussed near the end of this paper. For the Twente wiggler focussing forces are large and therefore errors in these will play a significant role in degradation of the electron beam properties. A measurement of focusing will also be discussed here.

The Twente wiggler [1] is designed to be used in two different configurations: one with a $6 \mathrm{MeV}$ electron beam and one with a $25 \mathrm{MeV}$ electron beam. In the first case overlap between the electron beam and the optical beam is of no concern since the optical beam is confined either by the waveguide or by gain guiding [2]. However in the 25 $\mathrm{MeV}$ case the overlap is important, so this criterion is applied to this wiggler. In general one must keep the center of the electron beam moving in a straight line, with a maximum deviation less than the amplitude of its wiggles, for good overlap between the electron beam and the optical beam.

If there is a strong focusing of the electron beam in the wiggler, i.e., there is more than one betatron oscillation over the length of the wiggler, the radius of the electron

\footnotetext{
* Corresponding author. Tel. +31 53 893971, fax +31 53 338065, e-mail j.w.j.verschuur@tn.utwente.n]
}

beam can be made constant throughout the wiggler with a radius smaller than the one required for weak focusing. The reduction in radius is proportional to the square root of the number of betatron cycles. This reduction is about a factor 3 for the $6 \mathrm{MeV}$ case and a factor 1 for the $25 \mathrm{MeV}$ case. An important additional effect of betatron focusing is that deviations in trajectories of the electrons caused by field errors are reduced because of the focusing forces. For the Twente wiggler the magnitude of the reduction is about equal to the factor of 3 mentioned above for field errors that extend beyond the length of one wiggler wavelength and less for localized errors. On the other hand, errors in the focussing field can destroy the quality of the electron beam. The conclusion is that attention has to be payed to focussing errors. In many cases wigglers are only tuned for the field on axis and not for possible focussing errors that are only present off axis. If the wiggler's gain is large. gain guiding occurs and all bets are off. At the moment we are not familiar with analyses of this problem. If the electron trajectory is curved, the light tends to follow around the curve. This tends to relax restrictions on wiggler accuracy. Since there is no reliable guidance in this case, we have attempted to adjust the wiggler to follow the restrictions given above, i.e., to make the wandering in the trajectories small compared to the amplitude of the wiggles.

\section{Field errors to be corrected}

By measuring the wiggler field, a new $\mathrm{mm}$ in the $x$ and $y$ directions, we can determine the average value of the field, as well as four of its gradients, $-\mathrm{d} B_{y} / \mathrm{d} y, \mathrm{~d} B_{y} / \mathrm{d} x, \mathrm{~d} B_{x} / \mathrm{d} y$, and $\mathrm{d} B_{x} / \mathrm{d} x$, where for measurements involving $B_{v}$ only the average value is considered. After tuning of the wiggler on 
axis we ended up with a wiggler with errors in three of the gradients seriously violating the criterion given above, and therefore they should be corrected. The four gradients are not equivalent to each other. Because the curl of $B_{z}$ is zero. the value of $\mathrm{d} B_{x} / \mathrm{d} y=\mathrm{d} B_{v} / \mathrm{d} x$, so focusing in the perpendicular directions has opposite sign. The new result is the transform of the original circular beam into an ellipse of the same area. The gradients $\mathrm{d} B_{x} / \mathrm{d} x$ and $\mathrm{d} B_{y} / \mathrm{d} y$ behave differently. If the gradients are large, the focusing effects become first order. This is the case for the Twente wiggler. however the gradients are small compared to the betatron focusing.

\section{The correction technique}

For a pure permanent wiggler adding small correction dipoles in the form of permanent magnets is a proven technique [3]. Modifying the field with small metal shims is a more convenient technique for hybrid wigglers, although the configuration of the shims is somewhat more complicated. The techniques are equivalent since the metal shims are polarized by the field and act as dipoles. These thin iron plates can only be efficiently placed on a pole face, well away from the center of the pole face. They can easily correct $\mathrm{d} B_{y} / \mathrm{d} x$ and $\mathrm{d} B_{x} / \mathrm{d} y$ errors. Since these two gradients are equal (with opposite sign), they should both be corrected by the same addition of two shims. Since a single shim also affects the field on axis. shims for correcting the off-axis field are usually placed on successive poles. The gradients $\mathrm{d} B_{v} / \mathrm{d} y$ and $\mathrm{d} B_{x} / \mathrm{d} x$ are not corrected as easy. This correction needs two shims on the same pole. The gradient that is produced by such a configuration is quite weak because of the nearness of the dipole placement to the horizontal symmetry axis. The gradient's strength is proportional to the $\sin (\theta)$, where $\theta$ is the angle between the horizontal plane and the line from the edge of the upper right pole face to the edge of the lower left pole face. For the Twente wiggler this angle is about $12^{\circ}$. If too many or too large shims are used on a single pole, a sextapole field may be generated in addition to a quadrupole field. The sextapole field will introduce an asymmetry in which the electrons with opposite displacements relative to the axis will be bent in the same direction instead of the opposite directions, as needed for focusing. The sextapole field can be cancelled by distributing the shims over several poles. In our case we did not have to go that far. We stopped correcting the quadrupole errors when the sextupole errors started to dominate the quadrupole errors.

\section{Experimental results}

As expected. the gradients $\mathrm{d} B_{y} / \mathrm{d} x$ and $\mathrm{d} B_{x} / \mathrm{d} y$ were easy to correct. Extensive shim sets were necessary to adjust the other two gradients. For example, we placed thick shims on four successive poles, staggering the shims on both sides of the axis on the upper poles and then on the lower ones. Because of the difficulty of adding these shims in an exactly uniform manner, such aggressive modifications unavoidably effected the field on axis and the other gradients. The final trimming was done by slightly adjusting the positions of the shims already in place. Moving them closer to the axis increased their effect.

After tuning the measurements are roughly in agreement with our criteria, i.e., the deviation from a straight line of the electron beam over the whole length of the wiggler is less than the wiggle amplitude. These results are about a factor of 5-10 better than the starting point of an only on-axis tuned wiggler.

\section{Wiggler focusing}

We measured the dependence of the strength of the wiggle motion on displacements in the $x$ and $y$ directions. Fig. 1 shows a plot of these measurements. For equal focusing, the curvatures of these measurements should be equal, and the sum should be easily related to the period of


Fig. 1. Wiggle amplitude as a function of displacement from the axis. 
the wiggler. Within the rather wide limits of accuracy of the measurements, we found that the curvatures were in the ratio $\frac{3}{2}$ and that the sum was $1.2 \pm 15 \%$ times larger than expected. This is in good agreement. The ratio of curvatures of $\frac{3}{2}$ indicates that a well matched beam will have a slightly elliptical shape, extending more in the horizontal plane than in the vertical plane. It is expected that this is not a serious problem for the performance of the FEL.

\section{Longitudinal phase errors}

If there are large deviations in the amplitude or phase of the wiggler field, the bunched electrons will advance or fall
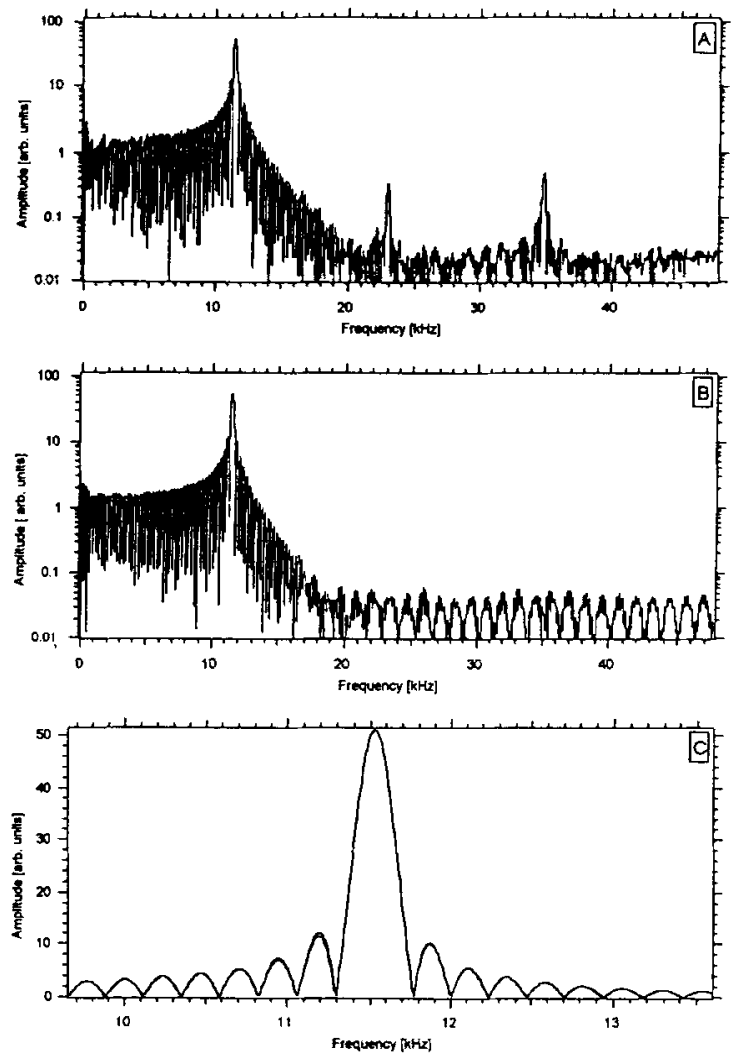

Fig. 2. Fourier transform of measured wiggler field (a). Ideal wiggler field connected to measured entrance and exit fields (b). An enlargement view of the central peak is also shown (c). back in phase relative to the phase of light they are interacting with. This will reduce the gain. To measure the magnitude of this effect. a Fourier transform of the first integral of the field is made and compared with a Fourier transform of a perfect sine wave with the same number of periods and terminated with the measured entrance and exit fields. Fig. 2 shows these transforms. The fact that in Fig. $2 \mathrm{c}$ the curves are almost perfectly superimposed shows that the phase errors are small. A significant phase error would have broadened the central peak and subsequently lowered its amplitude. The FWHM values of the peaks in the traces A, B are respectively 283.6 and 284.6 $\mathrm{Hz}$. We conclude from this that longitudinal phase errors are not of importance in this wiggler.

\section{Conclusions}

Correcting steering errors on axis only is by far not enough for a wiggler with strong focussing. A considerable improvement was made by correcting focussing errors with metal shims on the pole faces. It appeared to be sufficient to correct until the sextupole errors started to become stronger than the quadrupole errors. The focussing ratio, horizontal over vertical, of the wiggler was found to be $\frac{3}{2}$. This leads to a matched beam that is slightly elliptical. The longitudinal phase errors are qualified from a comparison between a Fourier transform of the measured field and an ideal field. The advantage of this method is that it treats the amplitude and wavelength errors simultaneously: No serious longitudinal phase errors were measured.

\section{References}

[1] J.W.J. Verschuur, G.J. Ernst and W.J. Witteman, Nucl. Instr. and Meth. A 318 (1992) 847

[2] G.J. Ernst and J.C. Goldstein, Nucl. Instr. and Meth. A 318 (1992) 649

[3] J.W.J. Verschuur, G.J. Ernst and W.J. Witteman. Nucl. Instr. and Meth. A 358 (1995) ABS85. 